\title{
Contribution phytoécologique et dynamique à l'étude des écosystèmes forestiers. Applications aux forêts du Nord-Est de la France
}

\author{
J.-C. RAMEAU \\ Professeur de Phytoécologie forestière à l'E.N.G.R.E.F. \\ Thèse de Doctorat d'Etat ès Sciences naturelles, septembre 1987
}

Cette thèse se compose de deux parties:

1 - Les principales publications parues de 1971 à 1987, présentées en plusieurs volets : phytosociologie forestière, dynamique de la végétation, cartographie de la végétation, typologie forestière, études dérivées (floristique, chorologie, phytogéographie, autécologie, patrimoines naturels, écologie et aménagements).

2 - Un rapport de synthèse rassemblant les réflexions sur les concepts et leur structuration, dans les domaines suivants: phytosociologie forestière, dynamique de la végétation et typologie forestière.

L'auteur souligne les aménagements à apporter à la démarche phytosociologique sigmatiste pour une meilleure prise en compte, d'une part des conditions écologiques à l'origine du déterminisme de la végétation (édaphiques, climatiques, biotiques, chrologiques, historiques $\rightarrow$ individualisation de races «écologiques » ou phytogéocœenoses, de sylvofaciés...) et d'autre part, des phénomènes dynamiques (stratégies adaptatives des espèces, phases ponctuant la vie d'une association végétale, diversification des modalités évolutives au sein d'une même station...).

La phytosociologie deviendrait ainsi plus fonctionnelle dans la caractérisation des unités écologiques fondamentales que sont les écosystèmes (unités issues de l'intégration à la phytogéocœnose, de la dynamique, du fonctionnement et des résultats de ce fonctionnement).

Les réflexions sur la dynamique conduisent l'auteur à préciser les différents comportements des essences : pionnières, postpionnières, dryades, nomades dans les cycles sylvigénétiques des forêts européennes. La place d'une essence dans une succession et sa participation à la phase optimale dépendent de ses propriétés biologiques (stratégies adaptatives), de ses exigences, de son optimum et de son amplitude écologiques, de son pouvoir dynamogénétique vis-à-vis des autres espèces. Après une étude de quelques exemples d'ontogenèse de climax, l'accent est mis sur les manteaux arborescents, phase pionnière de la sylvigenèse, très répandue en Europe du fait des facteurs historiques (longue série de défrichements et de déprises agricoles).

Une définition dynamique du concept de climax est proposée, avec, par ailleurs, la distinction de trois types de climax régionaux (climatiques, édaphiques et stationnels). Les essaims climaciques régionaux (N.E.) sont passés en revue avec définition des concepts de maturation sylvigénétique et de maturation floristique. Ensuite, l'auteur compare le degré de maturation sylvigénétique des principaux modèles forestiers français. Enfin, l'influence des phénomènes holocènes est mise en évidence sur les sylvigenèses actuelles (phylogenèse de la végétation forestière). 